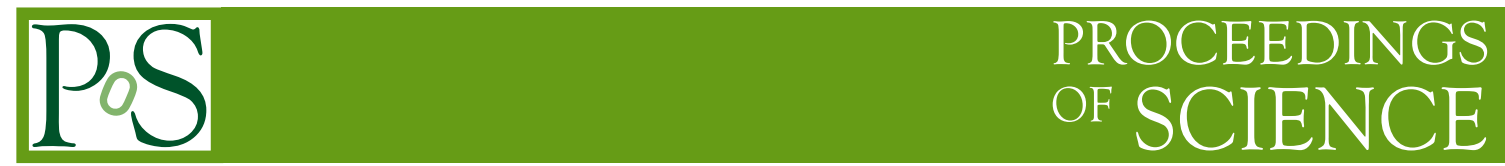

\title{
X-ray bursting neutron star atmosphere models
}

\author{
Valery Suleimanov* \\ IAAT, University of Tübingen, Germany \\ Kazan Federal University, Russia \\ E-mail: suleimanoveastro.uni-tuebingen.de

\section{Juri Poutanen} \\ University of Oulu, Finland \\ E-mail: juri.poutanen@oulu.fi \\ Mikhail Revnivtsev \\ Technische Universität München, Germany \\ Space Research Institute, Moscow, Russia \\ E-mail: m.revnivtsev@googlemail.com
}

\section{Klaus Werner}

IAAT, University of Tübingen, Germany

E-mail: wernereastro.uni-tuebingen.de

\begin{abstract}
We present an extended set of model atmospheres and emergent spectra of X-ray bursting neutron stars in low-mass X-ray binaries. Compton scattering is taken into account. The models were computed in LTE approximation for six different chemical compositions: pure hydrogen, pure helium, and solar mix of hydrogen and helium and various heavy elements abundances: $Z=1$, $0.3,0.1$, and $0.01 Z_{\odot}$. For each chemical composition the models are computed for three values of gravity, $\log g=14.0,14.3$, and 14.6, and for 20 values of relative luminosity $l=L / L_{\text {Edd }}$ in the range $0.001-0.98$. The emergent spectra of all models are fitted by the diluted blackbody in the redshifted $R X T E / \mathrm{PCA}$ band 3-20 keV and the corresponding values of color correction factors $f_{\mathrm{c}}$ as function of $l$ are presented. We also show how to use these dependencies to estimate the basic parameters of neutron stars.
\end{abstract}

Fast $X$-ray timing and spectroscopy at extreme count rates

February 7-11, 2011

Champéry, Switzerland

\footnotetext{
* Speaker.
} 


\section{Method}

Neutron stars (NSs) showing photospheric radius expansion X-ray bursts can be used to determine NS parameters, such as their radius $R$ and mass $M$ if the distance to the source is known (if, for example, a source is situated in the globular cluster, [1,2]). The relation between the observed normalization of the blackbody $K$ as fitted to the spectra and the ratio of $R$ to the distance during late burst phases is:

$$
K^{1 / 2}=\frac{R_{\mathrm{BB}}(\mathrm{km})}{D_{10}}=\frac{R(\mathrm{~km})}{f_{\mathrm{c}}^{2} D_{10}}(1+z),
$$

where $D_{10}$ is the distance in units $10 \mathrm{kpc}$, and $f_{\mathrm{c}}=T_{\mathrm{c}} / T_{\text {eff }}$ is the color correction factor. Therefore, during the cooling phases of X-ray bursts the dependence $K(t)$ reflects the variations of the $f_{\mathrm{c}}(t)$ only. We suggest to fit the observed $K^{-1 / 4}-F$ relation by the theoretical $f_{\mathrm{c}}-l$ relation, where $F$ is the bolometric observed flux. From this fit we can obtain two independent values: $A=\left(R(\mathrm{~km}) \times(1+z) / D_{10}\right)^{-1 / 2}$ and $F_{\text {Edd }} \propto L_{\text {Edd }} /\left((1+z) D_{10}^{2}\right)$. Combining these values we can obtain a relation between $M$ and $R$, which is independent on the distance and corresponds physically to the maximum possible observed effective temperature on a NS surface (the Eddington temperature)

$$
T_{\text {eff, } \infty}=\left(\frac{G M c(1+z)}{\sigma R^{2} k_{\mathrm{e}}}\right)^{1 / 4} \frac{1}{1+z}=6.4 \times 10^{9} F_{\mathrm{Edd}}^{1 / 4} A^{-1} \mathrm{~K} .
$$

Here $k_{\mathrm{e}}=0.2(1+X) \mathrm{cm}^{2} \mathrm{~g}^{-1}$ is the electron scattering opacity and $X$ is the hydrogen mass fraction. In order to use this method we need an extended set of theoretical $f_{\mathrm{c}}(l)$ curves. The existing models [3] do not provide enough accuracy. In this paper, we present a new set of models as well as the application of the method to one of the X-ray bursters.

\section{Results of atmosphere modeling}

We computed model atmospheres of X-ray bursting NSs subject to the constraints of hydrostatic and radiative equilibrium assuming planar geometry in LTE approximation with Compton scattering taken into account (see details of the code in [4,5]).

We calculated an extended set of NS model atmospheres with 6 chemical compositions (pure $\mathrm{H}$, pure $\mathrm{He}$, and solar $\mathrm{H} / \mathrm{He}$ mixture with $Z=1,0.3,0.1$ and $0.01 Z_{\odot}$ or $[\mathrm{Fe} / \mathrm{H}]=0,-0.5,-1$ and -2 ), three surface gravities $\log g=14.0,14.3$ and 14.6 , and twenty luminosities $L / L_{\text {Edd }}: 0.001$, $0.003,0.01,0.03,0.05,0.07,0.1,0.15,0.2,0.3,0.4,0.5,0.6,0.7,0.75,0.8,0.85,0.9,0.95$, and 0.98. Corresponding $T_{\text {eff }}$ were calculated from $L$ using $\log g$ and the chemical composition. The model emergent spectra were fitted by diluted blackbody spectra $F_{\mathrm{E}}=w B_{\mathrm{E}}\left(f_{\mathrm{c}} T_{\mathrm{Edd}}\right)$ using four slightly different procedures in the redshifted RXTE/PCA energy band $(3-20)(1+z) \mathrm{keV}$. Here $w \approx f_{\mathrm{c}}^{-4}$ is the dilution factor. The redshifts were calculated from $\log g$ assuming $M=1.4 M_{\odot}$. Results are presented in Figs. 1 and 2. See details in [6]. 

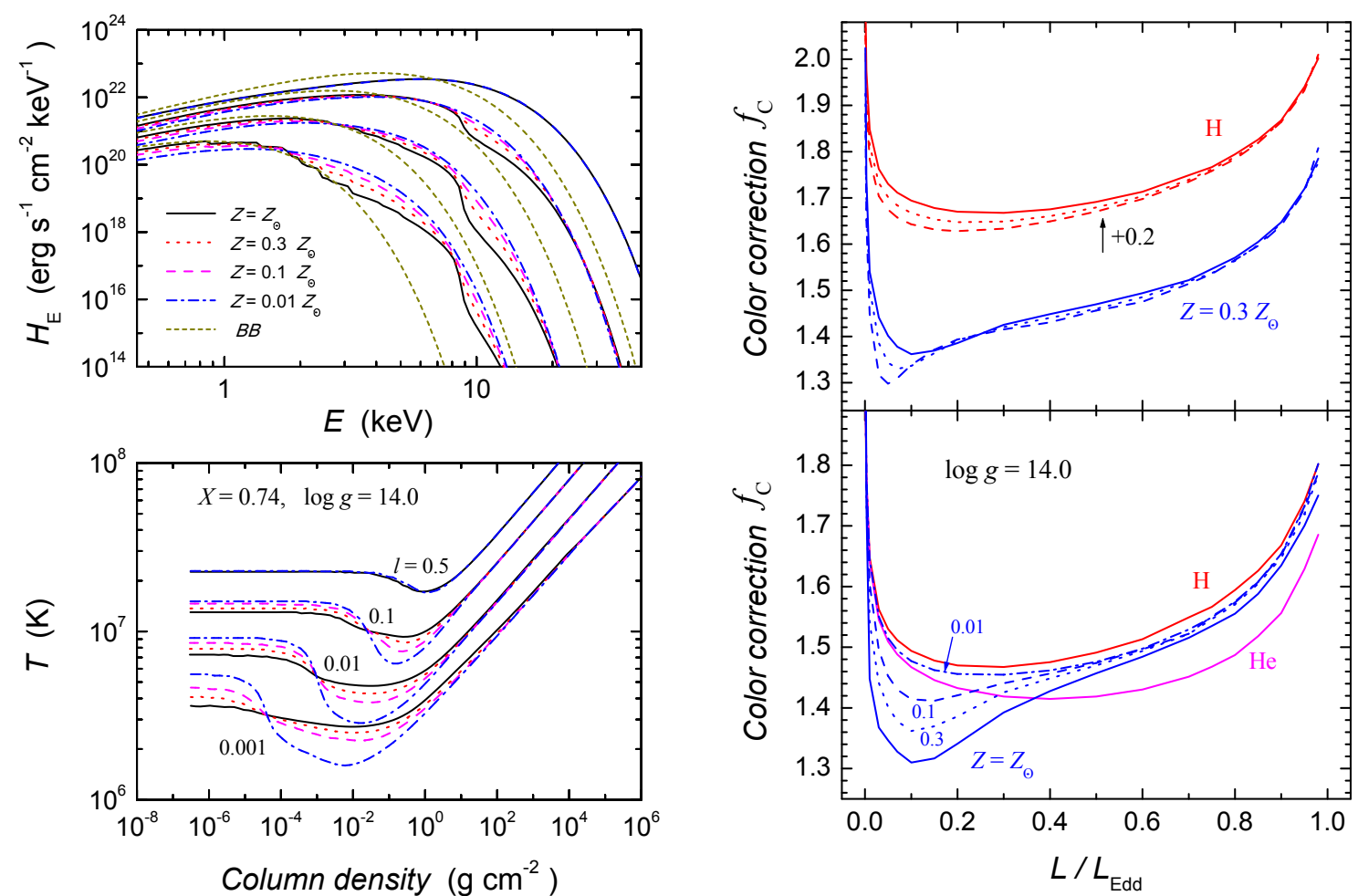

Figure 1: Left panels: Emergent (unredshifted) spectra (top panel) and temperature structures (bottom panel) of the model atmospheres with four relative luminosities $(l=0.5,0.1,0.01$ and 0.001$)$ and fixed surface gravity $(\log g=14.0)$ for solar hydrogen-helium mixture and various abundances of heavy elements: $Z / Z_{\odot}=1$ (solid curves), 0.3 (dash-dotted curves), 0.1 (dotted curves), 0.01 (dashed curves). In the top panel the blackbody spectra with effective temperatures are also shown by short-dashed curves. Right panels: Dependence of the color correction factors on the relative luminosity for various NS atmosphere models. Top panel: Dependences for hydrogen and solar H/He mixture with $Z=0.3 Z \odot$ models and different surface gravities $\log g=14.0$ (solid curves), 14.3 (dotted curves) and 14.6 (dashed curves). For clarity the dependences for hydrogen models are shifted by +0.2 . Bottom panel: Dependences for low gravity $(\log g=$ 14.0) models with various chemical compositions: pure hydrogen (upper curve), pure helium (lowest curve), and solar $\mathrm{H} / \mathrm{He}$ mixture with $Z / Z_{\odot}=1$ (dashed curve), 0.3 (dash-dotted curve), 0.1 (dotted curve), and 0.01 (solid curve). 


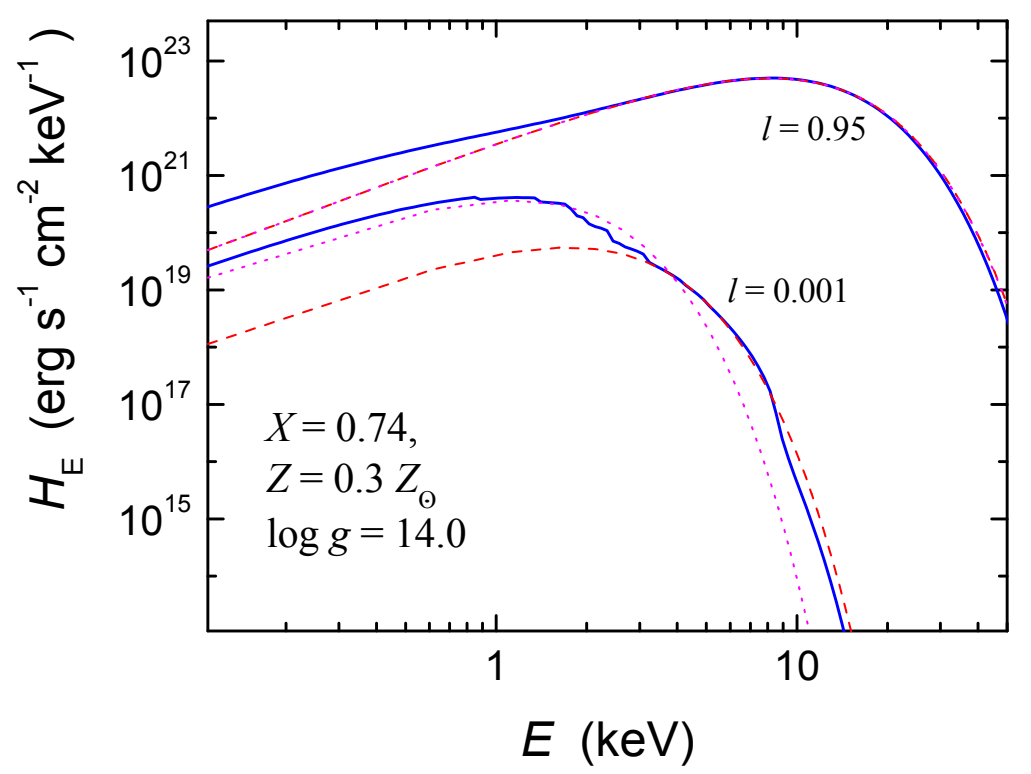

Figure 2: Examples of NS atmosphere spectra computed for solar $\mathrm{H} / \mathrm{He}$ mixture with $Z=0.3 Z_{\odot}$ for high $(l=0.95)$ and low $(l=0.001)$ luminosity and low gravity $(\log g=14.0)$. The computed spectra are shown by the solid curves, the fits with the blackbody with arbitrary dilution factor $w$ are shown by the dashed curves, and the fits with $w=f_{\mathrm{c}}^{-4}$ are shown by the dotted curves. The fits were performed in the 3-20 keV band.

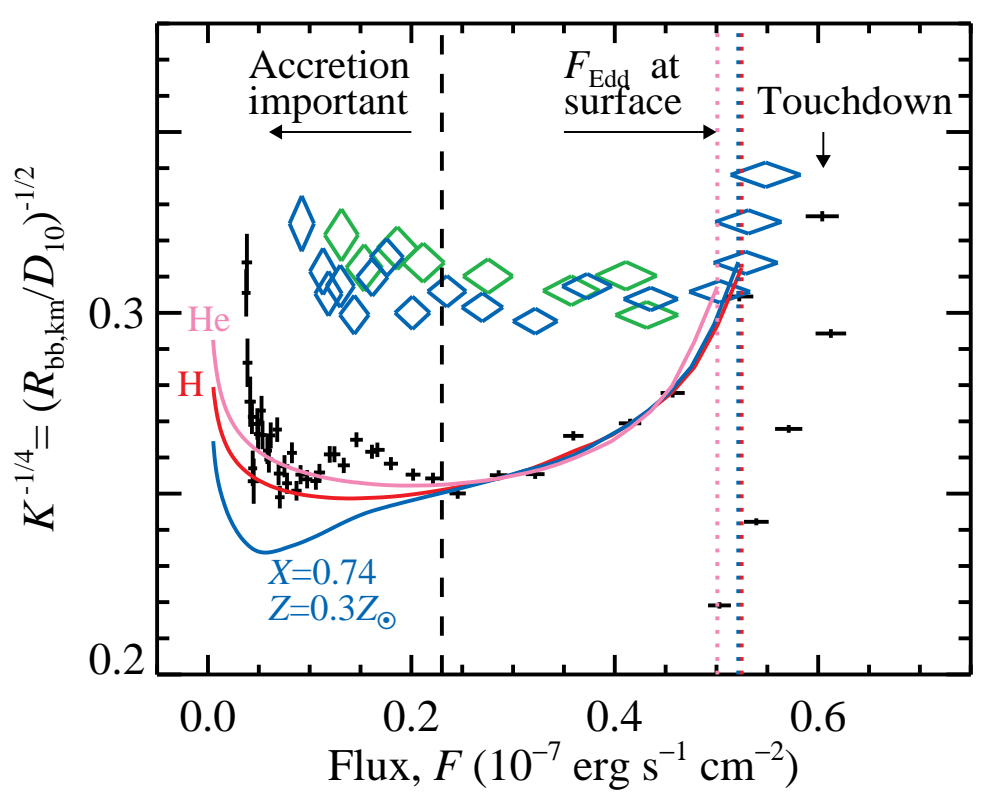

Figure 3: Comparison of the X-ray burst data for 4U 1724-307 to the theoretical NS atmosphere models. The crosses represent the observed dependence of $K^{-1 / 4}$ vs. $F$ for the long burst, while diamonds represent two short bursts. The solid curves correspond to the three best-fit theoretical models for various chemical compositions. 


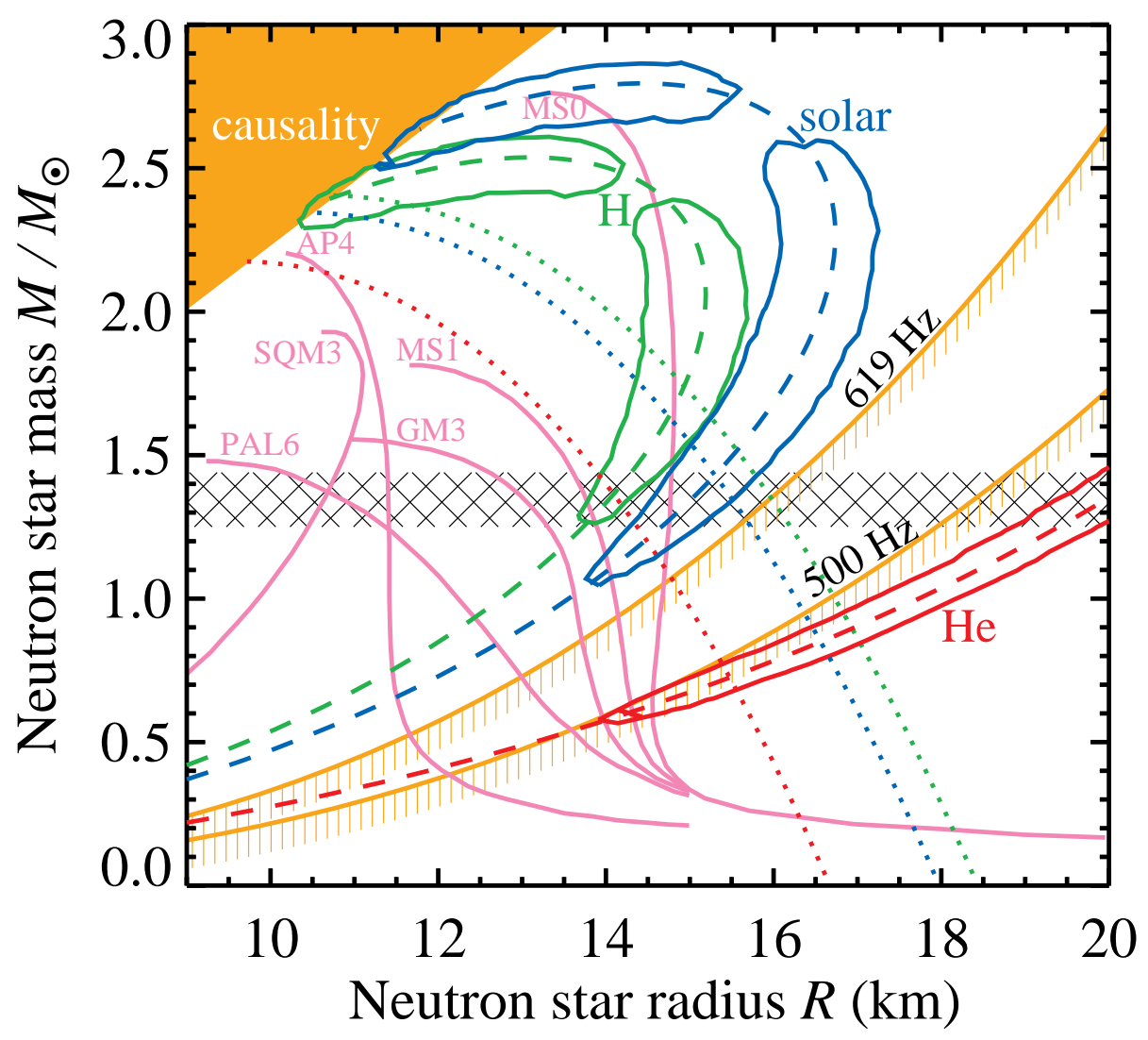

Figure 4: Constraints on mass and radius of the NS in 4U 1724-307 from the long burst spectra (fitted with the blackbody model and constant absorption). For a flat distribution of the distance between 5.3 and 7.7 $\mathrm{kpc}$ with Gaussian tails of $1 \sigma=0.6 \mathrm{kpc}$, the constraints are shown by contours ( $90 \%$ confidence level). The countours are elongated along the fixed Eddington temperature $T_{\mathrm{Edd}, \infty}$, which is shown by the dashed curves. The countours plotted correspond to the three chemical compositions: green for pure hydrogen, blue for the solar ratio of $\mathrm{H} / \mathrm{He}$ and subsolar metal abundance $Z=0.3 Z_{\odot}$ appropriate for Terzan 2 [8], and red for pure helium. The mass-radius relations for several equations of state of neutron and strange stars matter are shown by solid pink curves. The brown solid curves in the lower-right region correspond to the mass-shedding limit for various possible rotational frequencies.

\section{Application to 4U 1724-307 and conclusion}

We fitted the $K^{-1 / 4}-F$ relation as observed by RXTE [7] from the long burst of 4U 1724-307 on 1996 November 8 by the theoretical $f_{\mathrm{c}}-l$ relations (see Fig. 3). We obtained limits on $R$ and $M$ for various chemical compositions and the adopted distance between 5.3 and $7.7 \mathrm{kpc}$ with Gaussian tails of $1 \sigma=0.6 \mathrm{kpc}$ [8] (see Fig. 4 and [9] for more details). For H-rich compositions, the obtained $M$ and $R$ correspond to a stiff equation of state in the inner NS core. The atmospheres consisting of pure He are not acceptable.

Acknowledgements: The work is supported by the DFG grant SFB / Transregio 7 "Gravitational Wave Astronomy" (V.S.), Russian Foundation for Basic Research (grant 09-02-97013-rpovolzhe-a, V.S.), the Academy of Finland (grant 127512, J.P.), and DFG cluster of excellence "Origin and Structure of the Universe" (M.R.). 


\section{References}

[1] W. H. G. Lewin, J. van Paradijs, \& R. F. Taam, X-Ray Bursts, Space Science Reviews, 62, 223, (1993).

[2] F. Özel, T. Güver,\& D. Psaltis, The Mass and Radius of the Neutron Star in EXO 1745-248, ApJ, 693, 1775 (2009) [arXiv: 0810.1521 ].

[3] J. Madej, P. C. Joss, \& A. Różańska, Model Atmospheres and X-Ray Spectra of Bursting Neutron Stars: Hydrogen-Helium Comptonized Spectra, ApJ, 602, 904 (2004).

[4] V. Suleimanov, \& J. Poutanen, Spectra of the spreading layers on the neutron star surface and constraints on the neutron star equation of state, MNRAS, 369, 2036 (2006) [astro-ph / 0601689 ].

[5] V. Suleimanov, \& K. Werner, Importance of Compton scattering for radiation spectra of isolated neutron stars with weak magnetic fields, A\&A, 466, 661 (2007) [astro-ph/ 0702407 ].

[6] V. Suleimanov, J. Poutanen \& K. Werner, X-ray bursting neutron star atmosphere models: spectra and color corrections, A\&A, 527, A139 (2011) [arXiv: 1009.6147 ].

[7] S. V. Molkov, S. A. Grebenev, \& A. A. Lutovinov, An X-ray burst with strong photospheric radius expansion observed from the source $4 U$ 1724-307 in Terzan 2, A\&A, 357, L41 (2000) [astro-ph/0005082].

[8] S. Ortolani, E. Bica, \& B. Barbuy, V, I photometry of the metal-rich bulge globular cluster Terzan 2, A\&A, 326, 614 (1997).

[9] V. Suleimanov, J. Poutanen, M. Revnivtsev, \& K. Werner, Neutron star stiff equation of state derived from cooling phases of the X-ray burster $4 U$ 1724-307, ApJ, submitted [arXiv: $1004.4871]$. 